\title{
Guest editorial: web of things
}

\author{
Quan Z. Sheng ${ }^{1}$ Xue $\mathrm{Li}^{2}$ • Anne H.H. $\mathrm{Ngu}^{3}$ - Yongrui Qin ${ }^{4}$ - Dong $\mathrm{Xie}^{5}$
}

Published online: 8 July 2016

(C) Springer Science+Business Media New York 2016

\section{Introduction}

As indicated by the inventor of the World Wide Web, Tim Berners-Lee, "it isn't the documents which are actually interesting; it is the things they are about!". The last level of abstraction for the Web is to connect real things. Indeed, with the recent advances in radio-frequency identification (RFID) technology, wireless sensor networks, and Web services, the Web has emerged as the platform to connect billions of physical objects, i.e., "Web of Things" (WoT) (Sheng et al. 2011; Yao et al. 2015). WoT offers the capability of integrating the information from both the physical world and the virtual one, which creates tremendous business opportunities such as efficient supply chains, smart cities, and improved environmental monitoring.

Quan Z. Sheng

qsheng@cs.adelaide.edu.au

1 School of Computer Science, The University of Adelaide, Adelaide, 5005, SA, Australia

2 School of Information Technology and Electrical Engineering, University of Queensland, Brisbane, 4072, QLD, Australia

3 Department of Computer Science, Texas State University, San Marcos, TX, USA

4 School of Computing and Engineering, University of Huddersfield, Huddersfield, United Kingdom

5 Department of Computer Science and Technology, Hunan University of Humanities, Science and Technology, Hunan, China
While it is widely understood that WoT offers exciting capabilities to change the world and improve our life quality just as what the Web has done in the past 20 years, WoT also presents significant challenges (Qin et al. 2016). With billions of things interconnected and present over the Web, one inevitable challenge in the new era of WoT lies in how to efficiently and effectively manage things, which is critical for a number of important applications such as object discovery (e.g., finding a quiet restaurant), recommendation (e.g., suggesting a device that can consume a video stream), and mashup (e.g., composing device functionalities for a new service). In addition, massive volumes of real-time data ("big data") will be produced by these connected things and their associated sensors. Novel solutions are therefore required to effectively collect, process, analyze, and mine this big data. These solutions need to consider challenging issues of WoT data such as dynamicity, uncertainty, and high dimensionality.

In the rest of this article, we first discuss some specific research challenges around the Web of Things. We also present, in Section 3, some of the latest development in this research area. We particularly report three research papers addressing different aspects of the WoT.

\section{Web of things research challenges}

The Web of Things (WoT) raises a good number of research challenges (Matthew et al. 2013) and in this section, we wil discuss some of the most important ones.

The first challenge we would like to discuss is data quality and uncertainty. In WoT, as data volume increases, inconsistency and redundancy within data would become paramount issues. Fan et al. (2010) indicate that one of the central problems for data quality is inconsistency detection 
and when data are distributed, the detection would be far more challenging. This is because inconsistency detection often requires shipping data from one site to another. Meanwhile, inherited from radio frequency identification (RFID) data (Cao et al. 2011) and sensor data (Prabhakar and Cheng 2009), WoT data would be of great uncertainty, which also presents significant challenges. The second one is about Transaction Handling. WoT does not have a state which makes transaction handling a near impossibility. When the data being updated is spread across hundreds or thousands of nodes with differing update policies, it would be difficult to define what the transaction is. As (James et al. 2009) point out that the problem is that the world is changing fast, the data representing the world is on multiple nodes and existing database technology cannot manage. Techniques developed for streamed and real-time data may provide some directions.

The World Wide Web, and hence WoT, contains thousands of Frequently Updated Timestamped Structured (FUTS) data sources, such as sport scores, stock exchange information, weather updates, real-time flight details, auction prices and traffic reports. FUTS data sources represent states and updates of real-world things. There are three types of weakness of current technology when dealing with FUTS data sources (James et al. 2009): (i) there is no data management system that easily displays FUTS past data; (ii) there is no current efficient crawler or storage engine to collect and store FUTS data; and (iii) querying and delivering FUTS data is very limited. New data management systems are therefore needed with new user interfaces, parsers, storage engines and delivery mechanisms. In addition, WoT data will be increasingly distributed and mobile. Semantic meta-data can play a greater role in the structuring of knowledge contained in the Internet and could be used to facilitate efficient querying, increased personalization and data integration. Centralized data stores would not be suitable for a highly distributed, data intensive environment (James et al. 2009).

In a WoT environment, the physical space and the virtual (data) space co-exist, and interact simultaneously. To allow users to process and manipulate information seamlessly between the real and digital spaces, novel technologies must be developed (Ooi et al. 2009). Large amount of data and information must flow to/from co-space in order to ensure that the real and virtual worlds are synchronized. This brings new challenges such as a need to process heterogeneous data streams in order to materialize real world events in the virtual world and more intelligent processing to send interesting events in the co-space to someone in the physical world. Semantic technologies provide a promising future of the Web, where machines will be able to understand the data on the Web. The full potentials of WoT would heavily rely on the progress of semantic Web since things and machines should play a much more important role than human in WoT to process and understand data, and react to the real world. This calls for new research. For example, with the increasing efforts in building public knowledge bases (such as DBpedia, FreeBase, Linked Open Data Cloud, etc.), how these knowledge bases can be effectively used to add to the understanding of raw data coming from sensor data streams and other types of data streams via semantic enrichment is a great challenge. Further, considering the excessively large amount of subscriptions, to produce proper semantic enrichment to meet different enrichment needs from different subscribers also poses great challenges. Finally, how to incorporate semantic enrichment techniques with semantic event processing to provide much better expressiveness in event processing will also demand a large amount of research efforts.

Data mining aims to facilitate the exploration and analysis of large quantities of data, which can help to extract useful information for the huge amount of WoT data. Data mining challenges may include extraction of temporal characteristics from sensor data streams, event detection from multiple data streams, data stream classification, activity discovery and recognition from sensor data streams, clustering and table summarization in large data sets, mining large (data, information or social) networks, sampling, and information extraction from the Web. Knowledge discovery is the process of extracting useful knowledge from data. This is essential especially when connected things populate their data to the Web. (Weikum 2011) identify the following issues related to knowledge discovery in WoT: (i) automatic extraction of relational facts from natural-language text and multi-modal contexts; (ii) large-scale gathering of factual-knowledge candidates and their reconciliation into comprehensive knowledge bases; (iii) reasoning on uncertain hypotheses, for knowledge discovery and semantic search; and (iv) deep and real-time question answering, e.g., to enable computers to win quiz game shows.

Due to the proliferation of embedded devices in WoT, effective device security mechanisms are essential to the development of WoT technologies and applications. For example, RFID security presents many challenges. Potential solutions should consider aspects from hardware and wireless protocol security to the management, regulation and sharing of collected RFID data (Welbourne et al. 2009). (Lin et al. 2009) point out that establishing trust between a group of individuals remains a difficult problem. Besides, (Lagesse et al. 2009) argue that there is still no generic framework for deploying and extending traditional security mechanisms over a variety of pervasive systems. Regarding security concerns of the network layer, (Kounavis et al. 2010) suggest that the Internet can be gradually transformed to an information delivery infrastructure where all traffic is encrypted and authenticated. In fact, recent advances in 
the implementation of cryptographic algorithms can make general purpose processors capable of encrypting packets at line rates. Therefore, how to generalize such algorithms to WoT would be challenging as things in WoT normally only maintain low transmission rates and connections are usually lossy.

Privacy protection is a serious challenge in WoT. The fundamental problem is the lack of a mechanism to help people expose appropriate amounts of their identity information (Zhu and Zhu 2009). More and more personal devices such as mobile phones and multi-media players use embedded sensing. This means that people are wearing and carrying devices capable of sensing details about them such as their activity, location, and environment. Personal sensing can be used to detect their physical activities and hence bring about privacy concerns (Klasnja et al. 2009). In addition, temporal data are time-critical in that the snapshot at each timestamp must be made available in a timely fashion. However, due to the limited data, each snapshot likely has a skewed distribution on sensitive values, which renders classical anonymization methods not possible (Wang et al. 2010).

Finally, since WoT connects everyday objects to the Web, social concerns would become a hot topic in development of WoT. For example, home is a private and intimate place. It may have multiple stakeholders competing priorities and tolerances for what is acceptable and useful (Choe et al. 2011). To build smart homes based on WoT, similar social concerns should be considered. Further, online social networks with personal things information may incur social concerns as well, such as disclosures of personal activities and hobbies, etc. Appropriate economic and legal conditions and a social consensus on how the new technical opportunities in WoT should be used also represents a substantial task for the future (Mattern and Floerkemeier 2010).

\section{In this special issue}

This special issue aims at presenting the latest developments, trends, and research solutions for WoT technology, which received an enthusiastic response. Three papers were selected after several rounds of review by the guest editors and the invited reviewers. These papers cover some topics in WoT and related research that reflect some key directions in this active yet diverse research area.

The first paper, written by Sicari et al., "A securityand quality-aware system architecture for Internet of Things" (Sicari et al. 2016), focuses on the trustworthiness and quality of data collected and managed in the WoT environment. The authors propose a novel architecture that is able to support security, privacy and data quality requirements. The proposed architecture has the potential to satisfy users' and domain specific needs. Given that security, privacy, and data quality are the most critical issues around WoT, this research provides useful guidelines for the design of intelligent networked things.

The second paper by Stephan et al., "Semantic catalog of things, services, and data to support a wind data management facility" (Stephan et al. 2016), introduces a semantic catalog which is capable of making operations within a data management facility (DMF) more transparent. The semantic catalog leverages the recent advances in the Internet of Things (IoT), Semantic Web, Linked Services, Linked Open Data (LOD), and Resource Description Framework (RDF). The catalog can support catalog queries, as well as mashups. The design of this semantic catalog has been validated in a real-world wind characterization DMF for the U.S. Department of Energys Office of Energy Efficiency and Renewable Energy (EERE) Wind and Water Power Program. This DMF is capable of continuous data collection, processing, and preservation of in situ and remote sensing instrument measurements.

The third paper by Hossain, Quaddus, and Islam, "Developing and validating a model explaining the assimilation process of RFID: An empirical study" (Hossain et al. 2016), reports the authors' experience in their study of the assimilation process of radio frequency identification (RFID), which is a fundamental technology for WoT. The study involves 221 livestock farms in Australia that use RFID for livestock identification and tracing. Ten TechnologyOrganization-Environmental (TOE) factors on four stages of RFID assimilation processes have been carefully examined and the findings are discussed in the paper.

\section{Conclusion}

The Web of Things (WoT) is considered as a new revolution of the World Wide Web. In WoT, the possibility of seamlessly merging the real and the virtual worlds, through the massive deployment of embedded devices, opens up many new exciting directions for both research and development. The papers included in this special issue cover several topics and present some of the key directions in this vibrant and rapidly expanding area of research and development. We hope that the set of selected papers provides the community with a better understanding of the current directions and areas to focus in future, and inspires your own work.

Acknowledgments We thank all the authors for considering this special issue as an outlet to publish their research results in the area of WoT. We also would like to thank the referees who provided very useful and thoughtful feedback to the authors. Finally, we express our gratitude to the Editors-in-Chief, Professor Rao and Professor Ramesh for their kind support, advice, and encouragements throughout the preparation of this special issue. 


\section{References}

Cao, Z., Sutton, C., Diao, Y., \& Shenoy, P.J. (2011). Distributed inference and query processing for RFID tracking and monitoring. Proceedings of the VLDB Endowment, 4(5), 326-337.

Choe, E.K., Consolvo, S., Jung, J., \& Harrison, B.L. (2011). J. A. Kientz. Living in a Glass House A Survey of Private Moments in the Home. In Proceedings of the 13th International Conference on Ubiquitous Computing (Ubicomp). ACM, Beijing, China (pp. 4144).

Fan, W., Geerts, F., Ma, S., \& Müller, H. (2010). Detecting Inconsistencies in Distributed Data. In Proceedings of the 26th International Conference on Data Engineering (ICDE). IEEE, Long Beach, California, USA (pp. 64-75).

Hossain, M.A., Quaddus, M., \& Islam, N. (2016). Developing and validating a model explaining the assimilation process of RFID: An empirical study. Information Systems Frontiers, 18(4). doi:10.1007/s10796-014-9537-y.

James, A.E., Cooper, J., Jeffery, K.G., \& Saake. G. (2009). Research Directions in Database Architectures for the Internet of Things A Communication of the First International Workshop on Database Architectures for the Internet of Things (DAIT 2009). In Proceedings of the 26th British National Conference on Databases (BNCOD). springer, Birmingham, UK (pp. 225-233).

Klasnja, P.V., Consolvo, S., Choudhury, T., Beckwith, R., \& Hightower, J. (2009). Exploring Privacy Concerns about Personal Sensing. In Proceedings of the 7th International Conference on Pervasive Computing (Pervasive). Springer, Nara, Japan (pp. 176183).

Kounavis, M.E., Kang, X., Grewal, K., Eszenyi, M., Gueron, S., \& Durham, D. (2010). Encrypting the Tnternet. In Proceedings Of the ACM SIGCOMM conference on applications, technologies, architectures, and protocols for computer communications (SIGCOMM). ACM, New Delhi, India (pp. 135-146).

Lagesse, B., Kumar, M., Paluska, J.M., \& Wright, M. (2009). DTT A Distributed Trust Toolkit for Pervasive Systems. In Proceedings of the 7th Annual IEEE International Conference on Pervasive Computing and Communications (PerCom). IEEE, Galveston, TX, USA (pp. 1-8).

Lin, Y.-H., Studer, A., Hsiao, H.-C., McCune, J.M., Wang, K.-H., Krohn, M.N., Lin, P.-L., Perrig, A., Sun, H.-M., \& Yang, B.-Y. (2009). SPATE Small-group PKI-less Authenticated Trust Establishment. In Proceedings of the 7th International Conference on Mobile Systems, Applications, and Services (MobiSys). ACM, Kraków (pp. 1-14).

Mattern, F., \& Floerkemeier, C. (2010). From the Internet of Computers to the Internet of Things. In From active data management to Event-Based systems and more (pp. 242-259): Springer.

Matthew, S.S., Atif, Y., Sheng, Q.Z., \& Maamar, Z. (2013). The Web of Things: Challenges and Enabling Technologies Internet of Things and Inter-cooperative Computational Technologies for Collective Intelligence, Springer.

Ooi, B.C., Tan, K.-L., \& Tung, A.K. (2009). Sense The Physical, Walkthrough The Virtual, Manage The Co (existing) Spaces: A Database Perspective. SIGMOD Record, 38(3), 5-10.

Prabhakar, S., \& Cheng, R. (2009). Data Uncertainty Management in Sensor Networks. In Encyclopedia of Database Systems Springer US, USA (pp. 647-651).

Qin, Y., Sheng, Q.Z., Falkner, N.J., Dustdar, S., Wang, H., \& Vasilakos, A.V. (2016). When Things Matter A Survey on Data-Centric Internet of Things. Journal of Network and Computer Applications, 64, 137-153.
Sheng, Q.Z., Zeadally, S., Luo, Z., Jung, J.-Y., \& Maamar, Z. (2011). Ubiquitous RFID: Where are We?. Information Systems Frontiers, 12(5), 485-490.

Sicari, S., Cappiello, C., Pellegrini, F.D., Miorandi, D., \& CoenPorisini, A. (2016). A security-and quality-aware system architecture for Internet of Things. Information Systems Frontiers, 18(4). doi:10.1007/s10796-014-9538-x.

Stephan, E.G., Elsethagen, T.O., Berg, L.K., Macduff, M.C., Paulson, P.R., Shaw, W.J., Sivaraman, C., Smith, W.P., \& Wynne, A. (2016). Semantic catalog of things, services, and data to support a wind data management facility. Information Systems Frontiers, 18(4). doi:10.1007/s10796-015-9546-5.

Wang, K., Xu, Y., Wong, R., \& Fu, A.W.-C. (2010). Anonymizing Temporal Data. In Proceedings of the 10th IEEE International Conference on Data Mining (ICDM). IEEE, Sydney, Australia (pp. 1109-1114)

Weikum, G. (2011). Database Researchers: Plumbers or Thinkers?. In Proceedings of the 14th International Conference on Extending Database Technology (EDBT). ACM, Uppsala, Sweden (pp. 9 $10)$.

Welbourne, E., Battle, L., Cole, G., Gould, K., Rector, K., Raymer, S., Balazinska, M., \& Borriello, G. (2009). Building the internet of things using RFID the RFID ecosystem experience. IEEE Internet Computing, 13(3), 48-55.

Yao, L., Sheng, Q.Z., \& Dustdar, S. (2015). Web-based Management of the Internet of Things. IEEE Internet Computing, 19(4), 6067.

Zhu, F., \& Zhu, W. (2009). RationalExposure: a Game Theoretic Approach to Optimize Identity Exposure in Pervasive Computing Environments. In Proceedings of the 7th Annual IEEE International Conference on Pervasive Computing and Communications (PerCom). IEEE, Galveston, TX, USA (pp. 1-8).

Dr. Quan Z. Sheng is currently a full Professor in the School of Computer Science at the University of Adelaide. He holds a $\mathrm{PhD}$ degree in computer science from the University of New South Wales (UNSW) and did his post-doc as a research scientist at CSIRO ICT Centre. His main research interests include Web of Things, Internet of Things, big data analytics, Web services, Internet computing, and pervasive computing. He is the recipient of the ARC Future Fellowship (2014), Chris Wallace Award for Outstanding Research Contribution (2012), and Microsoft Research Fellowship (2003). He has more than 240 publications and is a member of the IEEE and the ACM.

Dr. Xue Li a full Professor in the School of Information Technology and Electrical Engineering at University of Queensland (UQ), Australia. He obtained the $\mathrm{PhD}$ degree in Information Systems from Queensland University of Technology (QUT) in 1997. From 1998 to 2000, he worked as a senior lecturer at University of New South Wales, Australia. Since December 2000, he has been working at UQ. Prof. Li's major areas of research interests and expertise include: data mining, RFID, Internet of Things, database Systems, and intelligent Web information systems. He has published more than 150 publications, most of them in premier international conferences and journal. He is a member of ACM, IEEE, and SIGKDD. 
Dr. Anne H.H. Ngu is a full Professor of the Department of Computer Science at Texas State University, USA. She has over 20 years of experience in research and development in IT with expertise in integrating data and applications, automating business processes on the Web, databases, object-oriented technologies and software agents. Prof. Ngu has worked in different countries as a researcher, including Institute of Systems Science in Singapore, Tilburg University in the Netherlands, Telcordia Technologies and MCC in Austin, Texas. From 1992-2000, Anne worked as a Senior Lecturer in the School of Computer Science and Engineering, University of New South Wales (UNSW). Prof. Ngu's research has been highly cited by her international peers. She is listed as one of the top 100 most cited authors in World Wide Web according to Microsoft Academic Search.

Dr. Yongrui Qin is a lecturer at School of Computing and Engineering, University of Huddersfield, UK. From Sep 2011 to Aug 2015, he was a PhD student researching on IoT data management and processing. Prior to that, he obtained his Master degree and Bachelor degree, both in Computer Science, from Fudan University, China. From Nov 2012 to May 2013, he was a Research Intern at DERI, Galway, Ireland (now The Insight Centre for Data Analytics). Yongrui's main research interests include Internet of Things, Web of Things, mobile computing, data mining, and data management. He has published more than 30 refereed technical papers, including publications in leading venues, such as IEEE Trans. on Parallel Distributed Systems, WWW Journal, IEEE Internet Computing, SIGIR, EDBT, and CIKM.
Dr. Dong Xie is an Associate Professor at the Department of Computer Science and Technology, Hunan University of Humanities, Science and Technology, China. He received a PhD degree in computer science from Central South University, Changsha, China in 2007. His research interests include Web of Things and data management with more than 40 research papers published. During 2011-2012, A/Prof. Xie worked at the School of Computer Science, the University of Adelaide as a visiting scholar, focusing on uncertainty management of RFID data. 